\title{
The development and validation of an age-structured model for the evaluation of disease control strategies for intestinal helminths
}

\author{
M. S. CHAN ${ }^{1 *}$, H. L. GUYATT², D. A. P. BUNDY ${ }^{*}$ and G. F. MEDLEY \\ ${ }^{1}$ WHO Collaborating Centre for the Epidemiology of Intestinal Parasitic Infections, Department of Biology, Imperial \\ College, London SW7 2BB, UK \\ ${ }^{2}$ Swiss Tropical Institute, Socinstrasse 57, CH 4002 Basel, Switzerland \\ ${ }^{3}$ Ecosystems Analysis and Management Group, Department of Biological Sciences, University of Warwick, \\ Coventry CV4 $7 A L, U K$
}

(Received 16 October 1993 ; revised 12 February 1994 ; accepted 12 February 1994)

\section{SUMMAR Y}

Epidemiological modelling can be a useful tool for the evaluation of parasite control strategies. An age-structured epidemiological model of intestinal helminth dynamics is developed. This model includes the explicit representation of changing worm distributions between hosts as a result of treatment, and estimates the morbidity due to heavy infections. The model is used to evaluate the effectiveness of different programmes of age-targeted community chemotherapy in reducing the amount of morbidity due to helminth infection. The magnitude of age-related heterogeneities is found to be very important in determining the results of age-targeted treatment programmes. The model was verified using field data from control programmes for Ascaris lumbricoides and Trichuris trichiura, and was found to provide accurate predictions of prevalence and mean intensities of infection during and following different control regimes.

Key words: intestinal nematodes, epidemiological model, age-targeted chemotherapy, evaluation of chemotherapy programmes, model verification.

\section{INTRODUCTION}

Intestinal helminth infections are amongst the most prevalent of human infections, affecting approximately one quarter of the world population (WHO, 1987; Bundy, 1990). The scale of morbidity caused by infections with the most prevalent species, Ascaris lumbricoides, Trichuris trichiura and hookworms (Ancyclostoma duodenale and Necator americanus) may amount to over 100 million (Chan et al. 1993). Although it has been observed that the prevalence of intestinal helminth infections drops following improvements in sanitation (Yokogawa, 1985), largescale improvements in sanitation are not considered likely in many developing countries in the short term. Community chemotherapy has been advocated as a possible immediate measure to reduce the morbidity associated with intestinal helminth infections (Warren et al. 1993; Savioli, Bundy \& Tomkins, 1992). Such chemotherapy programmes have two advantages: the reduction in worm burden and hence morbidity for the treated individuals and the reduction in further infection for all individuals (treated and untreated) due to the overall reduction in transmission. The rational planning of chemo-

* Present address : Department of Zoology, University of Oxford, South Parks Road, Oxford OX1 3PS. therapy programmes requires quantitative information on the likely costs and benefits of control. Since it is not always possible to fully test the control programmes in the field due to constraints on resources and time, epidemiological modelling, coupled with cost-effectiveness analysis, can provide a quantitative framework which can be used as a decision tool in conjunction with available data from field programmes (WHO, 1993).

Epidemiological models of both directly and indirectly transmitted helminth infections based on differential equation systems have been in existence for some time (reviewed by Anderson \& May, 1991; Woolhouse, 1991). However, the computational implementation of these models presents many difficulties due to the importance of parasite distributions in determining the dynamics of the system (Anderson \& Medley, 1985; Medley, Guyatt \& Bundy, 1993). Another modelling approach has sought to incorporate as many epidemiological and environmental processes as possible using stochastic microsimulation, as was done for the Onchocerciasis Control Program (Plaiser et al. 1990; Habbema et al. 1992). Although such models may give more accurate predictions within the local context for which they are developed, the empirical manner with which many processes are incorporated and the large number of local parameters required as inputs 
suggests that complex simulation models may have limited applicability when used outside of the originally intended locality or time-scale.

Recently, a computer-based epidemiological model to assess community chemotherapy programmes for intestinal helminths has been developed (Medley et al. 1993), based on the differential equation models of Anderson (1980). Novel features of this new model include a dynamically changing worm burden distribution which can accurately reflect the effects of control measures, estimation of morbidity due to heavy worm burdens and the linking of the model to a cost-effectiveness analysis using real cost data (Guyatt, Bundy \& Evans, 1993).

In this paper, we describe the development of an age-structured version of this model for $A$. lumbricoides, $T$. trichiura and schistosomiasis. For these species, age-intensity relationships have been consistently observed to be convex (Bundy, 1988; Anderson \& May, 1985). The highest intensity of infection is generally observed in school-age children who may also contribute relatively more to transmission due to unsanitary behaviour patterns. These observations suggest that a child-targeted approach to chemotherapy may be effective and may also have an economic advantage because children are more accessible due to attendance at school (Warren et al. 1993). We develop an age-structured model which allows the analysis of different strategies of agetargeted treatment and compare the output of the model to field data from actual control programmes for $A$. lumbricoides and $T$. trichiura.

\section{CONCEPTUAL FRAMEWORK}

The helminth life-cycle is conceptualized as a population of mature worms in human hosts and a population of free-living infective stages in the environment. The host population can become infected by contact with the infective stages and can contaminate the environment with infective stages. Unlike the Medley et al. (1993) model, in which there is only one group of hosts, the current model divides the host population into two groups : children and adults (Fig. 1). In the model, there are two differences between these groups. Children both acquire infections and contaminate the environment at a higher rate than adults. For both host groups, there is an overdispersed worm burden distribution such that most hosts harbour few worms and a few hosts have consistently higher worm burdens. These differential rates of infection acquisition are preserved during reinfection after treatment. Mass chemotherapy is simulated as instantaneous killing of worms. The coverage and frequency of treatment can be varied and treatment can be given to the whole population or to children only. The morbidity due to helminth infection is assumed to be associated with the worm burden by using a threshold worm burden above which morbidity is observed (Guyatt et al. 1990). The changes in mean worm burden, infection prevalence and prevalence of morbidity for both groups can be followed over several rounds of treatment.

\section{MODEL DEVELOPMENT}

The model is based on the basic model for directly transmitted helminths (Anderson, 1980; Anderson \& May, 1985, 1991) and implemented using the framework developed by Medley et al. (1993). Consider the host population divided into $n$ age groups with the $i^{\text {th }}$ group representing a proportion $\pi_{i}$ of the population. The worm population is divided into mature worms in the hosts and a pool of freeliving infective stages $(L)$. The host groups differ in two parameters, their contact rate with infective stages in the environment $\left(\beta_{i}\right)$ and their relative contribution to the pool of infective stages $\left(\lambda_{i}\right)$. These two parameters represent distinct processes. The contact rate $\left(\beta_{i}\right)$ is the rate of acquisition of infection (and subsumes the numerical size of the group) whereas the contamination rate $\left(\lambda_{i}\right)$ is related to the rate at which the individuals in the group contaminate the environment with their faeces. The mean worm burden of each group is denoted $W_{i}$. The model is illustrated for two groups (adults and children) in Fig. 1.

The rate of change of the mean worm burden of group $i$ can be represented by the following differential equation

$\frac{d W_{i}}{d t}=\beta_{i} L-\mu W_{i}$

where $\mu$ is the mortality rate of the mature worms.

The rate of change of infective stages can be described by

$\frac{d L}{d t}=\sum_{i} \lambda_{i} \pi_{i} f\left(W_{i}\right)-L \sum_{i} \beta_{i} \pi_{i}-\mu_{L} L$,

where $\mu_{L}$ is the mortality rate of infective stages and $f\left(W_{i}\right)$ is a density-dependent fecundity function (described below).

Since the life-span of the infective stages is much shorter than that of the mature worms in the hosts, the number of infective stages is assumed to be instantaneously adjusted to the equilibrium level $\left(L^{*}\right)$ (Anderson, 1980) which is given by

$L^{*}=\frac{\sum_{i} \lambda_{i} \pi_{i} f\left(W_{i}\right)}{\mu_{L}+\sum_{i} \beta_{i} \pi_{i}}$.

We define the basic reproductive number for each group $\left(R_{0 i}\right)$ to be the number of adult female parasites 


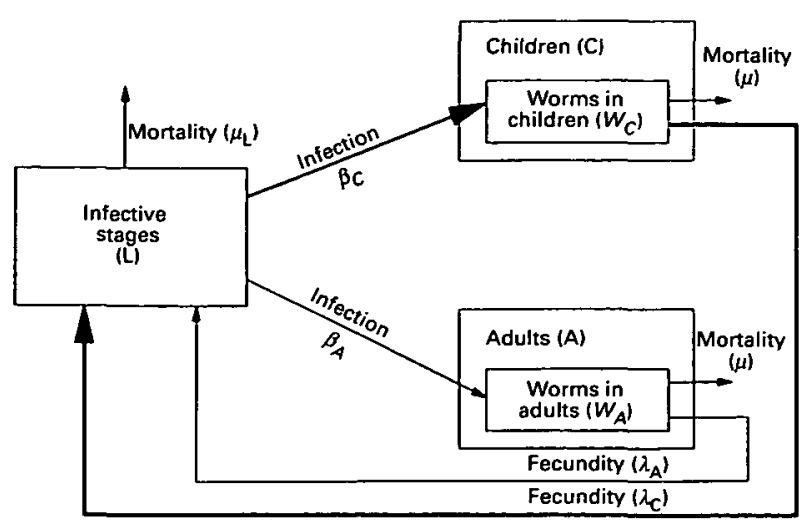

Fig. 1. Flowchart to show structure of a 2-age-group model. The host population is divided into children and adults with higher rates of infection acquisition and contamination for children (see text for details).

that arise in the total host population from 1 adult female in a host of group $i$ in the absence of densitydependent constraints. These quantities are given by

$R_{0 t}=\frac{\lambda_{i}}{\mu} \frac{\sum_{j} \beta_{j} \pi_{j}}{\mu_{L}+\sum_{j} \beta_{j} \pi_{i}}$.

The overall basic reproductive number $\left(R_{0}\right)$ for the whole parasite population is given as (Heesterbeek, personal communication)

$R_{0}=\frac{\sum_{i} \beta_{i} \lambda_{i} \pi_{i}}{\mu\left(\mu_{L}+\sum_{i} \beta_{i} \pi_{i}\right)}$.

After substitution of equations 3 and 4 into equation 1 , we obtain the following expression for the rate of change of mean worm burden in group $i$

$\frac{d W_{i}}{d t}=\beta_{i} \mu \frac{\sum_{j} R_{0 j} \pi_{j} f\left(W_{j}\right)}{\sum_{j} \beta_{j} \pi_{j}}-\mu W_{i}$.

By setting the differential to zero, the mean worm burden at equilibrium $\left(W_{i}^{*}\right)$ for group $i$ is given by

$W_{i}^{*}=\frac{\beta_{i} \sum_{j} R_{0 j} \pi_{j} f\left(W_{j}^{*}\right)}{\sum_{j} \beta_{j} \pi_{j}}$.

Note that this is not an explicit expression for the equilibrium since it appears on both sides of the equation. The value of the equilibria can therefore only be solved numerically.

To run the model numerically, it is necessary to specify the distribution of worms between hosts. The framework used to calculate the distributions is based on the model of Medley et al. (1993). To include the effects of predisposition to infection (Bundy \& Medley, 1992), the host population in each age group is divided into a number of hosts types, $g$, each with a susceptibility factor $h_{i g}$ and representing a proportion $\omega_{g}$ of the hosts in group $i$. The mean worm burden of hosts of type $g$ in group $i$ is given by $h_{i g} W_{i}$. The susceptibility factors and proportions are drawn from a gamma distribution with worm burdens within each host type distributed with a Poisson distribution. This gives an approximation to a negative binomial distribution at equilibrium which reflects distributions observed in endemic communities (Guyatt et al. 1990; Bundy \& Medley, 1992).

Given the above parasite distribution and assuming a negative exponential density-dependent fecundity function with parameter, $\gamma$, such that fecundity of a worm in a host with worm burden $W_{t}$ is $\lambda_{i} e^{-\gamma W_{i}}$, the overall effect of density dependence in group $i$ can be given by

$f\left(W_{i} ; k ; \gamma\right)=\underset{g}{W_{i g}} h_{i g} \omega^{-W_{i} h_{i g}\left(1-e^{-\gamma}\right)}$.

The value of the parameter $\gamma$ (assumed to be the same for all host groups) can be estimated from the equilibrium mean worm burden and the basic reproductive rate by the following iterative equation

$\sum_{i} R_{0 i} \pi_{i} f\left(W_{i}^{*} ; k ; \gamma\right)-\sum_{i} \pi_{i} W_{i}^{*}=0$.

The rest of the implementation of the model, including the simulation of chemotherapy treatment is identical to that described by Medley et al. (1993).

Morbidity is assumed to be associated with high worm burdens and a threshold worm burden $\left(T_{i}\right)$ is defined above which morbidity is observed. The effectiveness of a particular control programme is estimated by integrating the area between the morbidity time curve and the equilibrium mean morbidity level. This quantity represents the morbidity prevented by the control programme.

In this paper, a two-group model, with the groups representing children $(C)$ and adults $(A)$ is analysed. Children generally have both a higher rate of acquisition of infections and contribute relatively more to further infection; that is, the mean worm burden and the basic reproductive rate are both higher in children. Sensitivity analysis is carried out on the three new parameters in this model, the proportion of children in the population $\left(\pi_{c}\right)$, the relative mean worm burdens of adults and children (represented by the ratio $W_{A} / W_{C}$, or equivalently, $\beta_{A} / \beta_{C}$ ) and the relative basic reproductive rates of worms in adults and children (represented by the ratio $R_{0 A} / R_{0 C}$, or equivalently, $\left.\lambda_{A} / \lambda_{C}\right)$. Using parameters suitable for $A$. lumbricoides, the effectiveness of three age-targeted treatment strategies are compared : treatment of children yearly, treatment of children at 6-month intervals, and one population (children and adults) treatment followed by yearly treatment of children.

The output of the model is also compared with data from actual control programmes for $A$. lumbri- 


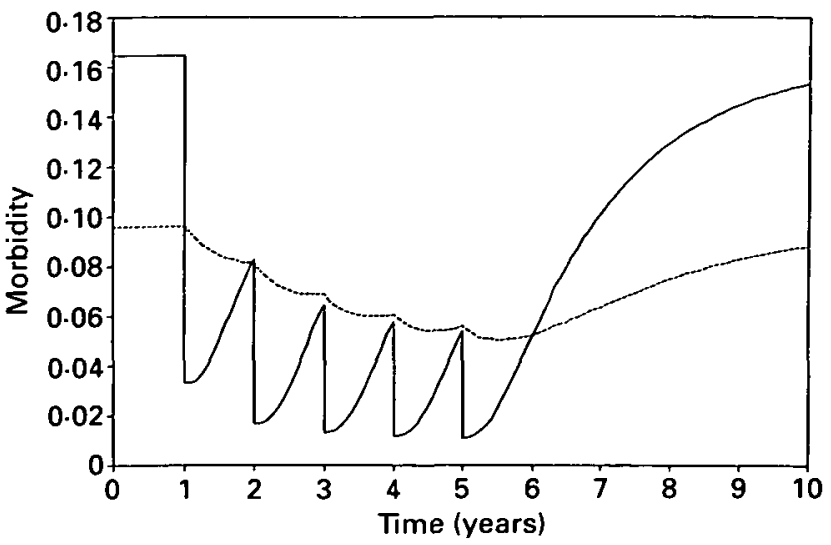

Fig. 2. An example of the results of a simulated chemotherapy programme. The prevalence of morbidity (estimated as the prevalence of infections with at least 20 worms) is plotted against time. ( $\longrightarrow$ ) Child morbidity; (---) adult morbidity. Children are treated at a coverage of $80 \%$ with a drug of efficacy $90 \%$ (drug kills $90 \%$ of worms) yearly from years 1 to 5 . Adults are not treated. The parameter values are, $W_{C}=10, W_{A}=7$, $R_{0 C}=3, R_{0 A}=1 \cdot 5, \pi_{C}=0 \cdot 25, \mu=1, k=0.543$.

coides in Myanmar (Burma) (Thein Hlaing et al. 1991) and T. trichiura in Montserrat (Bundy et al. 1990).

\section{RESULTS}

One example of the results of a simulated agetargeted treatment programme is shown in Fig. 2. In this simulation, children were treated during years 1-5 and adults were not treated at all. The figure shows the initial morbidity levels during the first year, the effect of the 5 annual treatments, and then the rebound in morbidity levels over the next 5 years. The morbidity (estimated as the prevalence of infections with at least 20 worms) is plotted over time separately for adults and children. When the treatment occurs, morbidity drops instantaneously in the children, but then rises again as they become reinfected. After the last treatment occurs, the morbidity slowly rebounds to the pre-control level after about 5 years. After each treatment, morbidity levels do not increase immediately and may even decrease initially. This is because of the overall reduction in transmission caused by the intervention. The figure also shows that treatment of only children can result in a significant reduction in morbidity for the adults. The benefit of the control programme in terms of morbidity reduction is estimated as the area between the morbidity curve and the equilibrium level. The benefit in terms of morbidity reduction is therefore greater in the children than in the adults. However, since the total population benefit is the sum of the benefits of each group weighted by the population in each group, and the population of adults is larger than that of the children, the benefit to untreated adults may be a significant proportion of the total community benefit of the chemotherapy
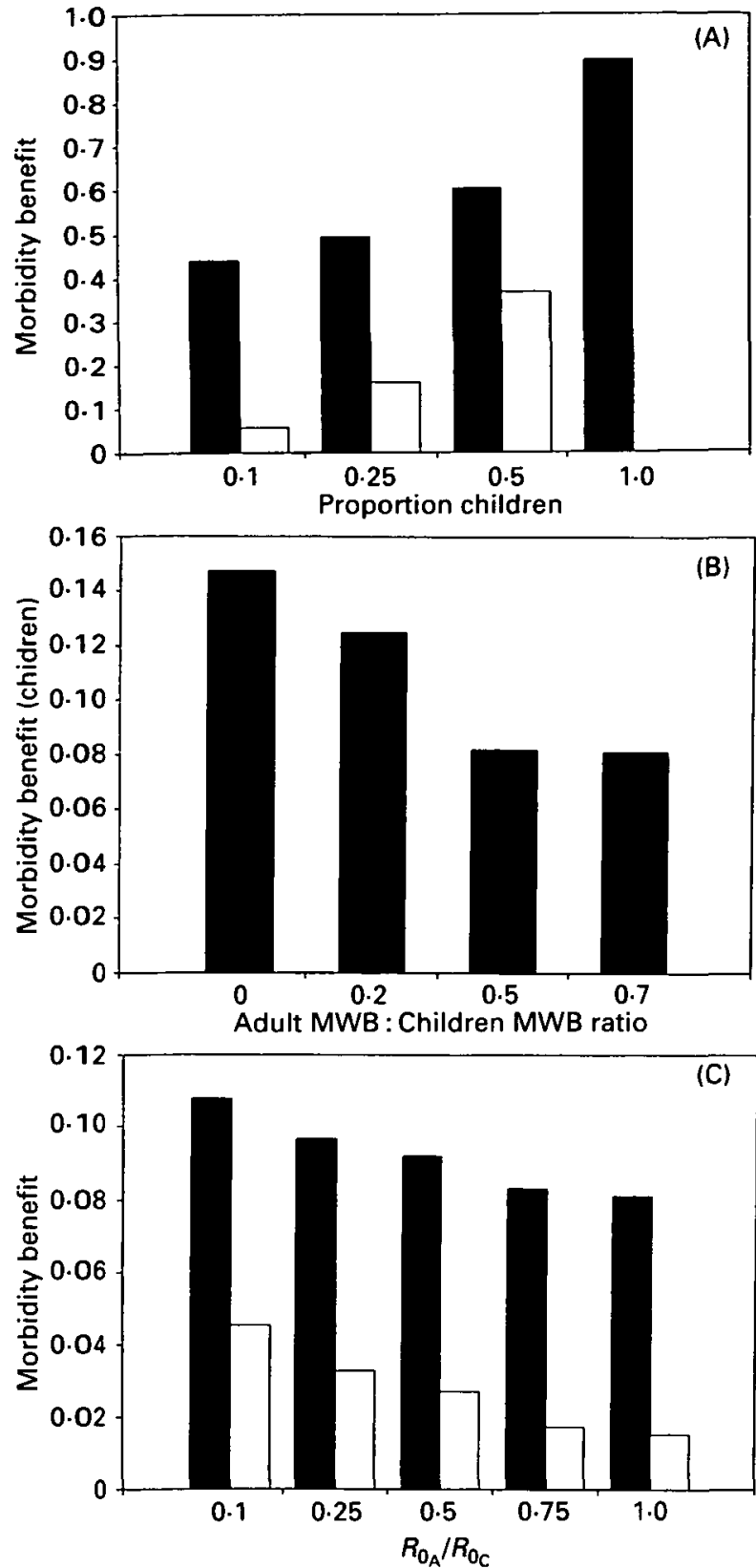

Fig. 3. Sensitivity analyses of the morbidity benefit of chemotherapy programmes to changes in the parameters of the model. Shaded bars represent the per capita benefit to children and empty bars the per capita benefit to adults. The default parameter set is $W_{C}=10, W_{A}=7$, $R_{0 C}=2, R_{0 A}=2, \pi_{C}=0.25, \mu=1, k=0.543$. The parameters varied in each graph are $(\mathrm{A}) \pi_{C}$, proportion of children in population; (B) $W_{A}$, equilibrium mean worm burden in adults; (C) $R_{0 A} / R_{0 C}$, ratio of reproductive rates of worms in adults and children.

programme, a quantity which cannot be estimated from the non-age-structured model.

The benefits of a chemotherapy programme will depend on the relative contributions of adults and children to transmission dynamics and community morbidity. Sensitivity analyses of the benefits to changes in model parameters relating to this are shown in Fig. 3. Figure $3 \mathrm{~A}$ shows the response of the benefits to changes in the proportion of children in 


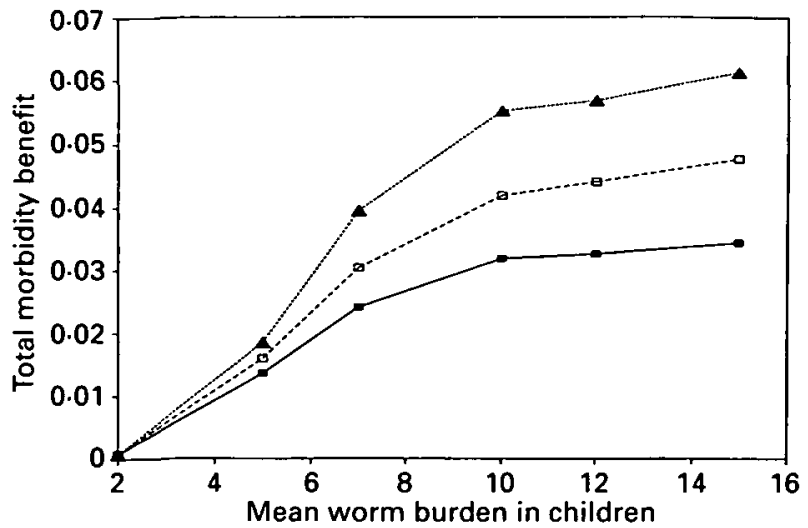

Fig. 4. Total community morbidity benefit for different treatment programmes plotted as a function of equilibrium mean worm burden in children (with the ratio $W_{A} / W_{C}$ kept constant). The strategies are (1) treatment of children yearly, years $1-5(\square)$; (2) treatment of children every 6 months, years 1-5 ( $\square$ ); (3) treatment of adults and children in year 1 and yearly treatment of children years $2-5(\boldsymbol{\Delta})$. The default parameter set is the same as in Fig. 3.

the population. The benefit of age-targeted chemotherapy to both children and adults is greater when the proportion of children is higher. This is an effect of coverage; when there are more children, a greater proportion of the population is treated. Figure $3 \mathrm{~B}$ shows the effect on children of changes in the equilibrium worm burden of adults. As the proportion of the worm population harboured by the adults increases, the benefits of age-targeted treatment are reduced, since the proportion of the worm population removed with child-targeted chemotherapy is relatively less. Likewise, if the relative contribution of adults to further transmission is increased (measured by the ratio $R_{0 A} / R_{o C}$ ), the benefit of child targeting to both children and adults is reduced (Fig. $3 \mathrm{C}$ ). Different contamination rates between children and adults may arise because children have different behaviour patterns and may be less likely to use sanitation facilities. These analyses highlight the importance of the scale of agerelated heterogeneities in the effects of age-targeted treatment. Two factors appear to be particularly important: the proportion of worms removed by treatment (changed when $\pi_{C}$ or $W_{A} / W_{C}$ are varied) and the reproductive potential of the remaining worms (changed when $R_{0 A} / R_{0 C}$ is varied).

The total morbidity benefit associated with different strategies of age-targeted treatment is shown in Fig. 4. The benefit is plotted as a function of the general level of transmission in a locality (measured as the equilibrium mean worm burden in children with the ratio $W_{A} / W_{C}$ constant) for the default strategy (yearly treatment of children) and two more intensive strategies (6-monthly treatment of children, and initial treatment of both adults and children followed by yearly treatment of children

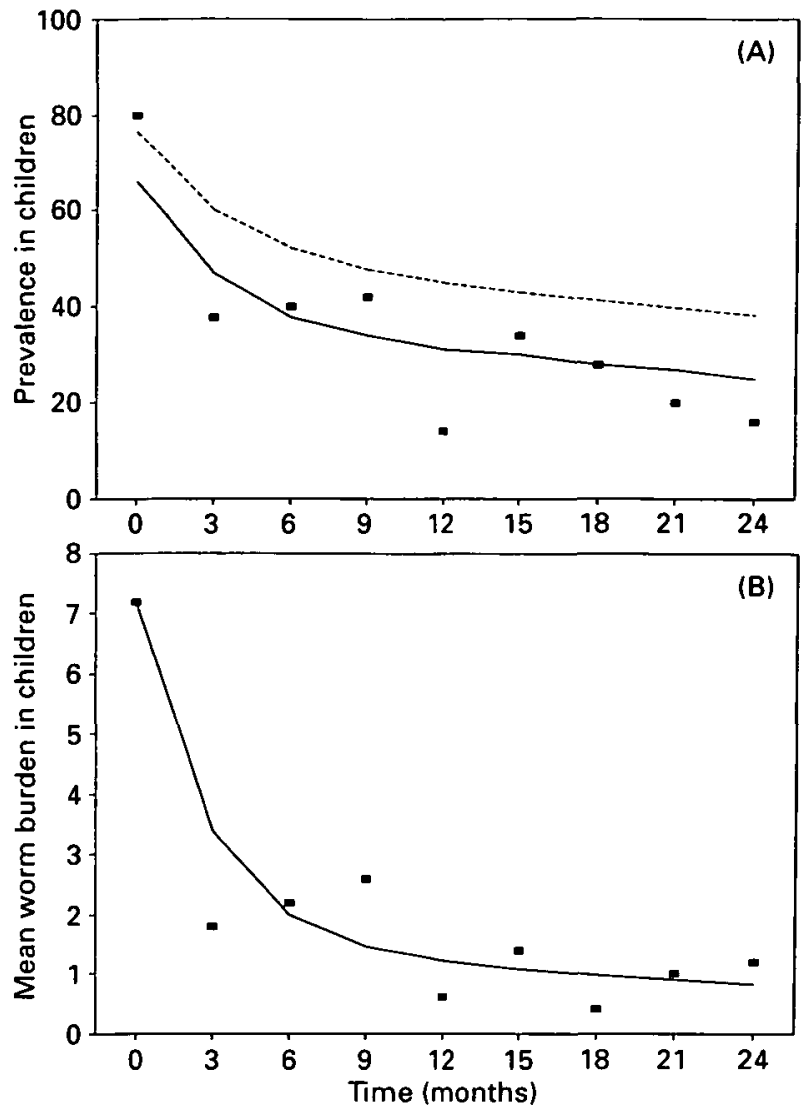

Fig. 5. Validation of model with field data for Ascaris lumbricoides infection from Thepya village, Myanmar (Burma). Data have been obtained from a study by Thein Hlaing et al. (1991) in which children were treated every 3 months throughout the study period. Parameters used for the model are the same as reported in the field study and are: $W_{C}=7 \cdot 2, W_{A}=2 \cdot 7$,

$R_{0 C}=1 \cdot 5, R_{0 A}=1 \cdot 5, \pi_{C}=0 \cdot 36, \mu=1, k=0.543$. (A) Prevalence of infection: ( $\square$ ) data points; (---) worm prevalence from the model; (-) prevalence from the model corrected to exclude male-only infections (correction term from Guyatt (1991). (B) Mean worm burden: ( $\square$ ) data points; ( $\longrightarrow$ ) model output.

only). For all strategies, intervention in a higher transmission area is always more effective. However, the benefit appears to flatten at very high levels of transmission perhaps due to the inability of the treatment to significantly reduce levels of transmission. Another observation is that a single initial treatment of adults and children followed by yearly treatment of children only, is more effective than 6monthly treatment of children at all levels of transmission. This is because the transmission potential of the whole population is reduced initially in the former strategy. In practice, the optimal strategy will depend on the relative costs of implementation as well as the effectiveness of control. In particular, the relative costs of treating adults and children will have a large influence on the choice of strategy.

The model was validated using data from an A. lumbricoides control programme in Myanmar 


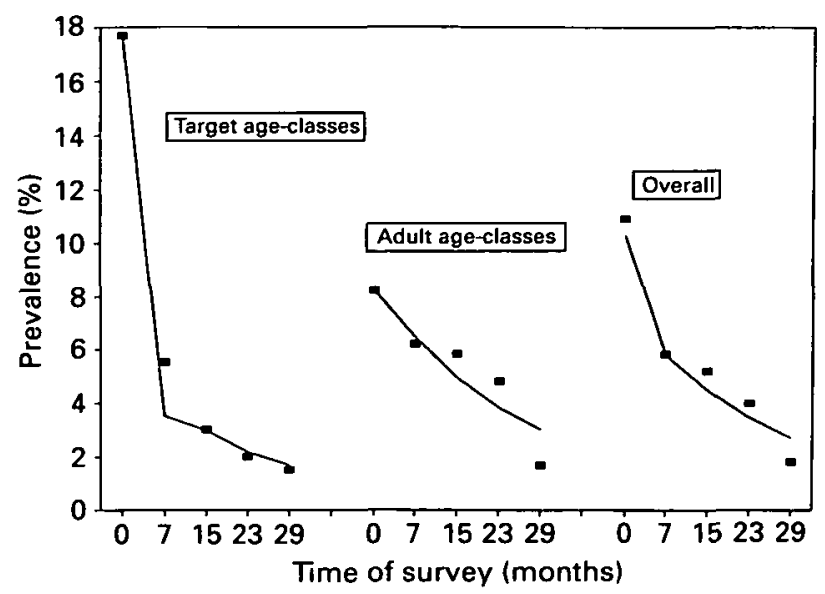

Fig. 6. Validation of model with field data for Trichuris trichiura infection from the island of Montserrat, West Indies. Data have been obtained from a study by Bundy et al. (1990) in which children were treated every 4 months throughout the study period. Parameters used for the model are the same as reported in the field study and are: $W_{C}=0 \cdot 3, W_{A}=0 \cdot 1, R_{0 C}=2, R_{0 A}=1$, $\pi_{C}=0 \cdot 22, \mu=1, k=0 \cdot 3$. The graph shows prevalence of infection over time for children and adults: $(\mathbb{W})$ data points; (-) model output.

(Thein Hlaing et al. 1991). The study was carried out in the village of Thepya (267 inhabitants). Children under 15 years old were treated every 3 months over a period of 2 years. Adults were not treated during the programme but adults found to be infected during the population survey at the end of the programme were given treatment. For model validation, parameter values are taken from the original data and the reproductive number $\left(R_{0}\right)$ is assumed to be equal in adults and children. The prevalence data are shown in Fig. 5A. The prevalences from the model (dashed line) appear to be higher than those from the data. However, the data are prevalences of positive egg counts, which excludes infections with only male worms (female A. lumbricoides produce eggs even in the absence of males). Using a correction to exclude male-only infections (from Guyatt, 1992; Guyatt \& Bundy, 1993), gives a much better fit to the prevalence data (solid line on Fig. $5 \mathrm{~A}$ ). The model gave a very good fit to the worm burden data (Fig. 5 B) which were estimated directly by anthelmintic expulsion. Unfortunately, prevalence in adults was not measured throughout the programme, so it was impossible to further verify the model for the estimated effect on adults. Similarly, although the assumption that the reproductive rate is equal in adults and children gives a good fit to the data, the possibility that the reproductive rates are different cannot be excluded due to the fact that data for adults are not available.

The model was also validated using data from a $T$. trichiura control programme on the island of Montserrat, West Indies (population 11500) (Bundy et al. 1990 and Bundy, unpublished data). At least
$90 \%$ of children aged $2-15$ years were treated every 4 months for over 2 years and, although treatment was offered to adults, coverage in adults was less than $4 \%$ for any treatment and it was assumed in the model that adults were not treated. After alternate treatment cycles, infection prevalence was estimated separately for target and non-target age groups in a random age-stratified sample of the population. Parameter values were again taken from the original data and it was assumed that the ratio of reproductive numbers $\left(R_{0 A} / R_{0 C}\right)$ was 0.5 . The results (Fig. 6) demonstrate convincingly that the model is able to predict the drop in prevalence over time in both the target and untreated groups.

\section{DISCUSSION}

The modelling framework of Medley et al. (1993) allowed the numerical implementation of the classical differential equation models developed by Anderson (1980) and Anderson \& May $(1985,1991)$ for intestinal helminths. This framework enables the practical value of different programmes of treatment by mass chemotherapy to be assessed. The model differs from more complex simulation models such as ONCHOSIM (Plaisier et al. 1990), for example, in that there are only a few input parameters and the results can be generalized to different environments. Usually, it will not be possible to assess all the environmental and epidemiological parameters before a programme is started, in which case, a general model such as the one presented here would be advantageous.

In this paper, the modelling framework of Medley et al. (1993) has been extended to include age-related heterogeneities in epidemiology and control. The analysis of the model shows that the epidemiological differences between the treated and untreated groups will have a large influence on the outcome of control. These differences include the rate of infection and the rate of contamination of the environment. If the untreated group harbours a large proportion of the parasites or makes a large contribution to the contamination of the environment, targeted treatment may not be an effective strategy. This may suggest that child targeting is least effective for the control of hookworm infections (Bundy, 1990) because hookworm infections are generally more prevalent in adults than children. Also, for control to be effective, the treated population should not be a very small proportion of the whole population. The results also show that the benefit of treatment to the untreated group may be a large proportion of the total community benefit and should be taken into account when evaluating the effectiveness of a programme. These analyses highlight the problems which may result from either modelling the whole population as one homogeneous unit or modelling the epidemiology in children as an isolated system. 
The model treats the population in terms of two age groups of constant size. The advantage of this approach is that these groups need not necessarily represent age groups and can be used for other types of groupings, for example by gender, ethnic origin or occupation. However, the use of constant groups for age omits the epidemiological effects of host maturation (transfer between groups). Given that the rate of maturation is small compared to the total adult population, and that the life-expectancy of parasites is relatively short (of the order of 1 year), the parasite distribution in the adult population can be assumed unaltered by the influx of children with a different parasite distribution. Other factors omitted in the model include worm mating probability, fluctuations in the population of infective stages, environmental fluctuations and acquired immunity. However, the fact that the model performs very well against data suggests that the quantitative effects of these omitted factors may not be very important.

Field studies have examined the epidemiological consequences of age-targeted treatment of children and observed reductions in adult infection prevalence and intensity (Thein Hlaing et al. 1991 ; Bundy et al. 1990). The model accurately predicted both data sets. The data suggest that the contamination rates of adults and children are about equal for $A$. lumbricoides infection in Myanmar and that the contamination rate of adults is half that of children for $T$. trichiura infection in Montserrat. These relative contamination rates are likely to vary between different localities but may be similar in the same locality for different species with the same transmission route. Although age-intensity curves are well described (Anderson \& May, 1985; Bundy, 1988 ), rates of contamination have not been well studied except for a few studies on schistosomiasis (Farooq \& Mallah, 1966; Cheesmond \& Fenwick, 1981). Differential rates of contamination may have a strong effect on the transmission dynamics and further research is needed on this.

These analyses have several implications for chemotherapy control programmes. If the epidemiology is modelled without age structure, for example, by only modelling the child population, one would tend to neglect the possible benefit to the untreated age group (adults) and overestimate the benefits to the treated age group (children). The age-structured model also highlights the importance of estimating the epidemiological parameters for both groups in the prediction of consequences of control. One parameter which may be important but is rarely measured is the relative contamination rate $\left(R_{0 A} / R_{0 C}\right)$. The age-structured model can also aid the selection of the suitable age group to target. Preliminary analysis of different age-targeted strategies suggests that an initial population treatment followed by child-targeted treatment may be more effective than simply increasing the frequency be- tween child-targeted treatments, although more information would be required on the cost implications of these approaches.

The model here can be expanded in several ways. One of the most interesting is the inclusion of explicit subpopulations of parasites. In the current model, the assumption of a single infective-stage population implies that the fraction of infective stages infecting a particular host group, $i$, which are produced in host group $j$ is determined by the rate of infective stage production in host group $j$. However, this proportional mixing assumption may be overly restrictive, and infective stages from one host group may be more or less likely to infect hosts in another group than would be expected proportionally. Future work in this area would develop the consequences of different mixing patterns on the population dynamics of parasite and host. In particular, such a framework would be essential when considering gene flow through parasite populations, for example, in the assessment of development of resistance or in the co-evolution of acquired immunity.

The detrimental effects of intestinal helminth infection have usually been considered as most important in children of school age due to effects on both physical (Bundy \& Cooper, 1989; Cooper, Bundy \& Henry, 1986; Cooper et al. 1990; Stephenson et al. 1989, 1990) and mental (Nokes et al. 1992) development. However, the epidemiological effects of child-targeted chemotherapy are not simple and have not been analysed quantitatively. This modelling study has presented a preliminary analysis of the epidemiological consequences of child-targeted chemotherapy and has suggested some general implications which would merit further investigation.

The authors would like to thank Roy Anderson, David Evans and Hans Heesterbeek for helpful discussions. D. A. P. Bundy thanks the Wellcome Trust for financial support. G. F. Medley is a Royal Society University Research Fellow. H. L. Guyatt is in receipt of a Wellcome Trust Travelling Fellowship. This study was supported by the Edna McConnell Clark Foundation.

\section{REFERENCES}

ANDERSON, R. M. (1980). The dynamics and control of direct life-cycle helminth parasites. Lecture Notes in Biomathematics 39, 278-322.

ANDERSON, R. M. \& MAY, R. M. (1985). Helminth infections of humans: mathematical models, population dynamics and control. Advances in Parasitology 24, 1-101.

ANDERSON, R. M. \& MAY, R. M. (1991). Infectious Diseases of Humans: Dynamics and Control. Oxford: Oxford University Press.

ANDERSON, R. M. \& MEDLEY, G. F. (1985). Community control of helminth infections of man by mass and selective chemotherapy. Parasitology 90, 629-60. 
BUNDY, D. A. P. (1988). Population ecology of intestinal helminth infections in human communities. Philosophical Transactions of the Royal Society of London B 321, 405-20.

BUNDY, D. A. P. (1990). Is the hookworm just another geohelminth? In Hookworm Disease, Current Status and New Directions, (ed. Schad, G. A. \& Warren, K. S.), pp. 147-64. London: Taylor \& Francis. BUNDY, D. A. P. \& COOPER, E. S. (1989). Trichuris and trichuriasis in humans. Advances in Parasitology 28, 107-73.

BUNDY, D. A. P. \& MEDLEY, G. F. (1992). Immunoepidemiology of human geohelminthiasis: ecological and immunological determinants of worm burden. Parasitology 104, S105-S119.

BUNDY, D. A. P., WONG, M. S., LEWIS, L. L. \& HORTON, J. (1990). Control of geohelminths by delivery of targeted chemotherapy through schools. Transactions of the Royal Society of Tropical Medicine and Hygiene 84, 115-20.

CHAN, M. S., MEDLEY, G. F., JAMISON, D. \& BUNDY, D. A. P. (1994). The evaluation of potential global morbidity attributable to intestinal nematode infections. Parasitology 109, 373-87.

CHEESMOND, A. K. \& FENWICK, A. (1981). Human excretion behaviour in a schistosomiasis endemic area of the Gezira, Sudan. Fournal of Tropical Medicine and Hygiene 84, 101-7.

COOPER, E. S., BUNDY, D. A. P. \& HENRY, F. J. (1986). Chronic dysentery, stunting, and whipworm infestation. Lancet ii, 280-1.

COOPER, E. S., BUNDY, D. A. P., MACDONALD, T. T. \& GOLDEN, M. H. N. (1990). Growth suppression in the Trichuris dysentery syndrome. European fournal of Clinical Nutrition 44, 285-91.

FAROOQ, M. \& MALLAH, M. B. (1966). The behavioural pattern of social and religious water contact activities in the Egypt 49 bilharziasis project area. Bulletin of the World Health Organization 35, 377-87.

GUYATT, H. L. (1992). Parasite population biology and the design and evaluation of helminth control programmes. Ph.D. thesis, University of London.

GUYATT, H. L. \& BUNDY, D. A. P. (1993). Estimation of intestinal nematode prevalence: influence of parasite mating patterns. Parasitology 107, 99-106.

GUYATT, H. L., BUNDY, D. A. P., MEDLEY, G. F. \& GRENFELL, B. T. (1990). The relationship between the frequency distribution of Ascaris lumbricoides and the prevalence and intensity of infection in human communities. Parasitology 101, 139-43.

GUYATT, H. L., BUNDY, D. A. P. \& EVANS, D. (1993). A population dynamic approach to the cost-effectiveness analysis of community-based anthelmintic treatment: Effects of treatment frequency. Transactions of the Royal Society of Tropical Medicine and Hygiene (in the Press).

habBema, J. D. F., ALley, E. S., Plaisier, A. P., VAN OORTMARSSEN, G. J. \& REMME, J. H. F. (1992).
Epidemiological modelling for onchocerciasis control. Parasitology Today 8, 99-103.

MEDLeY, G. F., GUYATT, H. L. \& BUNDY, D. A. P. (1993). A quantitative framework for evaluating the effect of community treatment on the morbidity due to ascariasis. Parasitology 106, 211-21.

NOKES, C., GRANTHAM-MCGREGOR, S. M., SAWYER, A. W., COOPER, E. S. \& BUNDY, D. A. P. (1992). Parasitic helminth infection and cognitive function in school children. Proceedings of the Royal Society of London B 247, 77-81.

Plaisier, A. P., VAN Oortmarssen, G. J., habbema, J. D. F., REMME, J. \& ALLEY, E. S. (1990). ONCHOSIM : A model and computer simulation program for the transmission and control of onchocerciasis. Computer Methods and Programs in Biomedicine 31, 43-56.

SAVIOLI, L., BUNDY, D. A. P. \& TOMKINS, A. M. (1992). Intestinal parasitic infections: a soluble public health problem. Transactions of the Royal Society of Tropical Medicine and Hygiene 86, 353-4.

STEPHENSON, L. S., LATHAM, M. C., KURZ, K. M., KINOTI, S. N. \& BRIGHAM, H. (1989). Treatment with a single dose of albendazole improves growth of Kenyan schoolchildren with hookworm, Trichuris trichiura and Ascaris lumbricoides infections. American Yournal of Tropical Medicine and Hygiene 41, 78-87.

STEPHENSON, L. S., LATHAM, M. C., KINOTI, S. N., KURZ, K. M. \& BRIGHAM, H. (1990). Improvements in physical fitness of Kenyan schoolboys infected with hookworm, Trichuris trichiura and Ascaris lumbricoides following a single dose of albendazole. Transactions of the Royal Society of Tropical Medicine and Hygiene 84, 277-82.

THEIN HLAING, THAN-SAW \& MYAT-LAY-KYIN (1991). The impact of three monthly age-targeted chemotherapy on Ascaris lumbricoides infection. Transactions of the Royal Society of Tropical Medicine and Hygiene 85 , 519-22.

WARREN, K. S., BÚNDY, D. A. P., ANDERSON, R. M., DAVIS, A. R., HENDERSON, D. A., JAMISON, D. T., PRESCOTT, N \& SENFT, A. (1993). Helminth infections. In Disease Control Priorities in Developing Countries, (ed. Jamison, D. T., Mosley, W. H., Measham, A. R. \& Bobadilla, J. L.), pp. 131-160. Oxford: Oxford University Press.

WORLD HEALTh ORganization (1987). Prevention and Control of Intestinal Parasitic Infections. WHO Technical Report Series No. 749. Geneva.

WORLD HEALTH ORGANIZATION (1993). Epidemiologica modelling for schistosomiasis control. American Fournal of Tropical Medicine and Hygiene (in the Press).

WOolhouse, M. E. J. (1991). On the application of mathematical models of schistosome dynamics. 1. Natural transmission. Acta tropica 49, 241-70.

YOKOGAWA, M. (1985). JOICFP's experience in the control of ascariasis within an integrated programme. In Ascariasis and its Public Health Significance, (ed. Crompton, D. W. T., Nesheim, M. C. \& Pawlowski, Z.S.), pp. 265-78. London: Taylor \& Francis. 